\title{
Exact two-terminal reliability of some directed networks
}

\author{
Christian Tanguy \\ CORE/MCN/OTT \\ France Telecom Division R\&D \\ 38-40 rue du Général Leclerc, 92794 Issy-les-Moulineaux Cedex 9, France. \\ christian.tanguy@orange-ftgroup.com
}

\begin{abstract}
The calculation of network reliability in a probabilistic context has long been an issue of practical and academic importance. Conventional approaches (determination of bounds, sums of disjoint products algorithms, Monte Carlo evaluations, studies of the reliability polynomials, etc.) only provide approximations when the network's size increases, even when nodes do not fail and all edges have the same reliability $p$.

We consider here a directed, generic graph of arbitrary size mimicking real-life long-haul communication networks, and give the exact, analytical solution for the two-terminal reliability. This solution involves a product of transfer matrices, in which individual reliabilities of edges and nodes are taken into account. The special case of identical edge and node reliabilities ( $p$ and $\rho$, respectively) is addressed. We consider a case study based on a commonly-used configuration, and assess the influence of the edges being directed (or not) on various measures of network performance. While the two-terminal reliability, the failure frequency and the failure rate of the connection are quite similar, the locations of complex zeros of the two-terminal reliability polynomials exhibit strong differences, and various structure transitions at specific values of $\rho$.

The present work could be extended to provide a catalog of exactly solvable networks in terms of reliability, which could be useful as building blocks for new and improved bounds, as well as benchmarks, in the general case.
\end{abstract}

\section{INTRODUCTION}

Network reliability has long been a practical issue, and will remain so for years, since networks have entered an era of Quality of Service (QoS). IP networks, mobile phone networks, transportation networks, electrical power networks, etc., have become "commodities." Connection availability rates of $99.999 \%$ are a goal for telecommunication network operators, and premium services may be deployed if the connection reliability is close enough to one. Reliability is therefore a crucial parameter in the design and analysis of networks.

The study of network reliability has led to a huge body of literature, starting with the work of Moore and Shannon [1], and including excellent textbooks and surveys [2], [3], [4], [5], [6], [7], [8]. In what follows, we consider a probabilistic approach, in which the network is represented by an undirected graph $G=(V, E)$, where $V$ is a set of nodes (also called vertices) and $E$ is a set of undirected edges (or links), each of which having a probability $p_{n}$ or $p_{e}$ to operate correctly. Failures of the different constituents are assumed to occur at random, and to be statistically independent events. Among the different measures of reliability, one may single out the $k$ terminal reliability, namely the probability that a given subset $K$ of $k$ nodes $(K \subset E$ ) are connected. The most common instances are the all-terminal reliability $\operatorname{Rel}_{A}(K \equiv E)$ and the two-terminal reliability $\operatorname{Rel}_{2}(s \rightarrow t)$, which deals with a particular connection between a source $s$ and a destination $t$. Both of them are affine functions of each $p_{n}$ and $p_{e}$.

The sheer number of possible system states, namely $2^{|E|+|V|}$, clearly precludes the use of an "enumeration of states" strategy for realistic networks, and shows that the final expression may be extremely cumbersome. Consequently, most studies have considered graphs with perfect nodes $\left(p_{n} \equiv 1\right)$ and edges of identical reliability $p$; radio broadcast networks have also been described by networks with perfectly reliable edges but imperfect nodes [9], [10]. It was shown early on - see for instance the discussion in [4], [11] - that the calculation of $k$-terminal reliability is \#P-hard in the general case, even with the restricting assumptions that (i) the graph is planar (ii) all nodes are perfectly reliable (iii) all edges have the same reliability $p$. All reliabilities are then expressed as a polynomial in $p$, called the reliability polynomial.

The difficulty of the problem has stimulated many approaches: partitioning techniques [12], sum of disjoint products [13], [14], [15], [16], [17], [18], graph simplifications (series-parallel reductions [1], delta-wye transformations [19], [20], [21], factoring [22]), determination of various lower and upper bounds to the reliability polynomial [2], [4], [23], [24], [25], Monte-Carlo simulations [26], [27], [28], and ordered binary decision diagram (OBDD) algorithms [29], [30], [31], [32]. Other decomposition methods have also been proposed [33], [34], [35]. The reliability polynomial has been extensively studied [36], [37], [38], with the aim of finding general information from the structure of its coefficients [36], [37] or the location of its zeros in the complex plane [39].

In recent years, the tremendous growth of Internet traffic has called for a better evaluation of the reliability of connections in optical networks. Actual failure rates and maintenance data show that a proper evaluation of two-terminal reliabilities must put node and edge equipments on an equal footing, i.e., both edge (fiber links, optical amplifiers) and node (optical crossconnects, routers) failures must be taken into account. The possibility of node failure has been considered in early papers [9], [40]. Adaptation of algorithms to include imperfect nodes 
has been addressed [32], [41], [42], [43], [44]. In order to be realistic, different edge reliabilities should be used too: for instance, the failure rate of optical fiber links is often assumed to increase with their length.

In recent works, we have shown that the two- and allterminal reliabilities can be exactly calculated for recursive network architectures, where the underlying graphs are undirected and the edge/node reliabilities arbitrary [45], [46], [47]. The final expressions are products of transfer matrices, each element of which is a multilinear polynomial of the individual edge or node reliabilities constituting the 'building block' (or 'elementary cell') of the recursive graph.

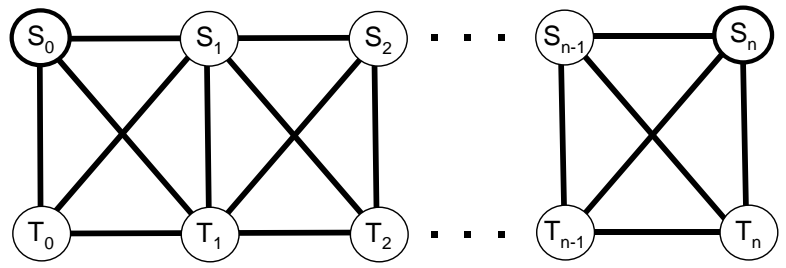

Fig. 1. General $K_{4}$ ladder. The source is always $S_{0}$, the destination is $S_{n}$ or $T_{n}$.

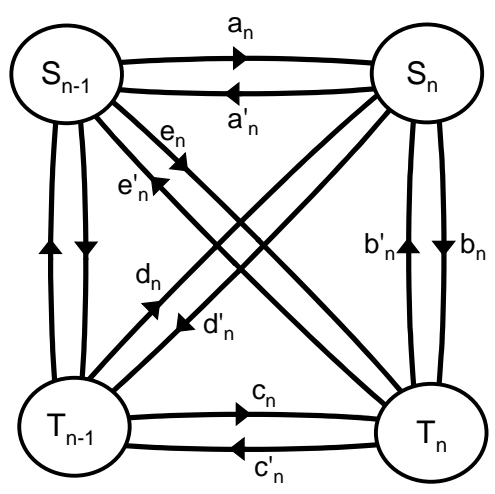

Fig. 2. Last building block of the directed $K_{4}$ ladder. Edges and nodes are identified by their reliabilities.

In this work, we show this general result holds for directed networks too. As an example, we calculate the twoterminal reliability of the directed $K_{4}$ ladder displayed in Fig. 1. This network describes a common (nominal + backup paths) architecture, with additional connections between transit nodes enabling the so-called "local protection" policy, which bypasses faulty intermediate nodes and/or edges. By letting the individual node and edge reliabilities take arbitrary values, we actually do not add to the complexity of the problem but make the internal structure of the problem more discernible. It is then easier to fully exploit the recursive nature of the graph. The two-terminal reliability's exact expression is a product of $5 \times 5$ transfer matrices, as in the undirected case [47]; consequently, it can also be determined for an arbitrary size (length) of the network. If edges have the same reliability $p$, and nodes the same reliability $\rho$, the two-terminal reliability can be expressed as a sum over the eigenvalues of the unique transfer matrix, and its generating function is a rational fraction. For large networks, the eigenvalue of highest modulus is, to all purposes, the scaling factor of the asymptotic power-law behavior. The determination of the failure frequency and the failure rate of the connection is then straightforward. Prompted by the nearly universal character of the Brown-Colbourn conjecture [39], we also address the location of complex zeros of the twoterminal reliability polynomial and show that they (i) may be quite different for directed and undirected networks (ii) exhibit structural transitions at various values of $\rho$.

Our aim is (i) to give a description of the methodology followed in the derivation of the final results, so that researchers or engineers involved in reliability studies can use them, even in worksheet applications (ii) emphasize again the importance of algebraic structures of the underlying graphs in the determination of network reliability [5], [48].

Our paper is organized as follows. In Section II, we give the basic formula and methodology used in the decomposition method [47], which must be adapted here for directed graphs. In Section [III we give the exact solution for the two-terminal reliability for the directed $K_{4}$ ladder (with source $S_{0}$ and destination $S_{n}$ or $T_{n}$ ). In Section IV] we consider directed and undirected configurations of a special architecture. We first give the generating functions of the two-terminal reliabilities when all edges and nodes reliabilities are $p$ and $\rho$, respectively; we then derive very simple analytical expressions for $\operatorname{Rel}_{2}$. The average failure frequency and the failure rate of the connection under consideration are then deduced. We then show that the location of the complex zeros of $\mathrm{Rel}_{2}$ differ in the two configurations. We conclude by proposing several directions in which the present results may be extended.

\section{GRAPH DECOMPOSITION}

The purpose of our method is to simplify the graph by removing links of the $n^{\text {th }}$ (last) elementary cell of the network, namely the edges and nodes indexed by $n$, a procedure called pivotal decomposition or deletion-contraction [4]. In the case of undirected graphs [47], we saw that if the end terminal $t$ (which can be regarded as perfect) is connected to node $u$ through edge $e$, with respective reliabilities $p_{u}$ and $p_{e}$, then

$$
\begin{aligned}
\operatorname{Rel}_{2}(G)= & \left(1-p_{e}\right) \operatorname{Rel}_{2}(G \backslash e)+p_{e} p_{u} \operatorname{Rel}_{2}(G \cdot e) \\
& +p_{e}\left(1-p_{u}\right) \operatorname{Rel}_{2}(G \backslash u),
\end{aligned}
$$

where $G \backslash e$ and $G \backslash u$ are the graphs where $e$ or $u$ have been deleted, and $G \cdot e$ the graph where $t$ and $u$ have been merged through the "contraction" of $e$; eq. (1) merely sums probabilities of disjoint events. Here, we must adapt this decomposition to a directed graph. This is done very easily by first discarding all edges whose origin is $t$ and then applying eq. (1) to the remaining graph. This procedure, along with standard series-parallel reductions, has to be repeated for the three secondary graphs in order to take advantage of the structural recursivity of the graph. After a finite number of such reductions, we get replicas of the original graph, albeit with one less elementary cell and with the $(n-1)^{\text {th }}$ cell's edge and node reliabilities possibly renormalized by those of the $n^{\text {th }}$ cell, or set to either zero or one. In order to ensure the existence 
of a recursion relation, the graph structure must be closed under successive applications of eq. (1); it may initially require the use of extra edges with symbolic reliabilities, so that all nodes of an elementary cell are connected pair-wise, even if such links do not exist in the graph under consideration (the "scaffolding principle"). At this point, a recursion hypothesis is needed, giving for instance $\operatorname{Rel}_{2}\left(S_{0} \rightarrow S_{n}\right)$ as a sum over specific polynomials in the reliabilities indexed by $n$; these are often obvious from the $n=2$ value. Going from $n-1$ to $n$ provides the transfer matrix linking the prefactors of the polynomials, because $\mathrm{Rel}_{2}$ is an affine function of each component reliability; the (often trivial) $n=1$ case serves as the initial condition of the recurrence.

\section{EXACT SOLUTION FOR THE DIRECTED $K_{4}$ LADDER}

Let us first illustrate this method by calculating $\mathcal{R}_{n}=$ $\operatorname{Rel}_{2}\left(S_{0} \rightarrow S_{n}\right)$ for the directed $K_{4}$ ladder shown in Fig. 1, a more detailed view of the last building block is displayed in Fig. 2. Following the guidelines of the preceding section, we remove the irrelevant edges, namely those indexed by $a_{n}^{\prime}$, $b_{n}^{\prime}$, and $d_{n}^{\prime}$. The first application of eq. (1) is represented in Fig. 3, where each prefactor is put in front of the associated secondary graph. Note that the three secondary graphs are structurally identical, they differ by the actual values of the edge reliabilities only. Clearly, a second application of eq. (1) provides two-terminal reliabilities, with $S_{n-1}$ or $T_{n-1}$ as new endpoints. This should call for a similar decomposition of the two-terminal reliability $\operatorname{Rel}_{2}\left(S_{0} \rightarrow T_{n}\right)$, after which we would get coupled recursion relations for the two destinations $S_{n}$ and $T_{n}$. However, this is unnecessary because the two destinations are identical under the permutations $a_{n} \leftrightarrow e_{n}, c_{n} \leftrightarrow d_{n}$ (and the corresponding $a_{n}^{\prime} \leftrightarrow e_{n}^{\prime}$, etc.), and $S_{n} \leftrightarrow T_{n}$. It turns out that $\mathcal{R}_{n}$ may be expressed as the sum of five polynomials in $a_{n}, \ldots, T_{n}$ (see below). This is also true for $\operatorname{Rel}_{2}\left(S_{0} \rightarrow T_{n}\right)$, which leads us to a $10 \times 10$ transfer matrix (the calculations are routinely performed by mathematical software). Because several lines of this matrix are identical, regrouping terms actually allows to limit the transfer matrix's dimension to 5, as in the undirected case [47]. The value of $\mathcal{R}_{1}$, which can be easily calculated, leads to

$$
\mathcal{R}_{n}=\left(\begin{array}{lllll}
1 & 0 & 0 & 0 & 0
\end{array}\right) M_{n} M_{n-1} \cdots M_{1} M_{0}\left(\begin{array}{l}
1 \\
0 \\
0 \\
0 \\
0
\end{array}\right) ;
$$

for $\operatorname{Rel}_{2}\left(S_{0} \rightarrow T_{n}\right)$, the left vector should be $\left(\begin{array}{lllll}0 & 1 & 0 & 0 & 0\end{array}\right)$. The transfer matrix $M_{i}$ is given by

$$
M_{i}=\left(\begin{array}{ccccc}
x_{1} & x_{2} & x_{3} & x_{4} & x_{5} \\
x_{6} & x_{7} & x_{8} & x_{9} & x_{10} \\
x_{18} & x_{17} & x_{14} & -x_{4}-x_{9} & -x_{5}-x_{10} \\
x_{20} & x_{19} & x_{15} & -x_{9} & -x_{10} \\
x_{11} & x_{12} & x_{13} & -x_{4} & -x_{5}
\end{array}\right) \text {, }
$$

with

$$
\begin{aligned}
& x_{1}=S_{i}\left(a_{i}+b^{\prime}{ }_{i} e_{i} T_{i}-a_{i} b_{i}^{\prime} e_{i} T_{i}\right), \\
& x_{2}=S_{i}\left(d_{i}+b^{\prime}{ }_{i} c_{i} T_{i}-b^{\prime}{ }_{i} c_{i} d_{i} T_{i}\right) \text {, } \\
& x_{3}=S_{i}\left(a_{i} d_{i}+a_{i} b^{\prime}{ }_{i} c_{i} T_{i}-a_{i} b^{\prime}{ }_{i} c_{i} d_{i} T_{i}+b^{\prime}{ }_{i} c_{i} e_{i} T_{i}\right. \\
& \left.-a_{i} b_{i}^{\prime} c_{i} e_{i} T_{i}+\left(1-a_{i}\right) b_{i}^{\prime}\left(1-c_{i}\right) d_{i} e_{i} T_{i}\right) \text {, } \\
& x_{4}=\left(1-a_{i}\right)\left(1-b_{i}^{\prime}\right) c_{i}^{\prime} d_{i} e_{i} S_{i} T_{i} \text {, } \\
& x_{5}=a_{i}\left(1-b_{i}^{\prime}\right) c_{i}\left(1-d_{i}\right) e_{i}^{\prime} S_{i} T_{i}, \\
& x_{6}=\left(e_{i}+a_{i} b_{i} S_{i}-a_{i} b_{i} e_{i} S_{i}\right) T_{i} \text {, } \\
& x_{7}=\left(c_{i}+b_{i} d_{i} S_{i}-b_{i} c_{i} d_{i} S_{i}\right) T_{i}, \\
& x_{8}=\left(c_{i} e_{i}+a_{i} b_{i} c_{i} S_{i}+a_{i} b_{i} d_{i} S_{i}-a_{i} b_{i} c_{i} d_{i} S_{i}\right. \\
& \left.-a_{i} b_{i} c_{i} e_{i} S_{i}+\left(1-a_{i}\right) b_{i}\left(1-c_{i}\right) d_{i} e_{i} S_{i}\right) T_{i} \text {, } \\
& x_{9}=a_{i}\left(1-b_{i}\right) c_{i} d^{\prime}{ }_{i}\left(1-e_{i}\right) S_{i} T_{i}, \\
& x_{10}=a^{\prime}{ }_{i}\left(1-b_{i}\right)\left(1-c_{i}\right) d_{i} e_{i} S_{i} T_{i} \text {, } \\
& x_{11}=\left(1-a_{i}\right)\left(1-b_{i}^{\prime}\right) e_{i} S_{i} T_{i} \text {, } \\
& x_{12}=\left(1-b^{\prime}{ }_{i}\right) c_{i}\left(1-d_{i}\right) S_{i} T_{i} \text {, } \\
& x_{13}=\left(1-b^{\prime}{ }_{i}\right)\left(a_{i} c_{i}-a_{i} c_{i} d_{i}+c_{i} e_{i}-a_{i} c_{i} e_{i}\right. \\
& \left.+\left(1-a_{i}\right)\left(1-c_{i}\right) d_{i} e_{i}\right) S_{i} T_{i}, \\
& x_{14}=\left(1-b^{\prime}{ }_{i}\right)\left(a_{i} c_{i}-a_{i} c_{i} d_{i}+c_{i} e_{i}-a_{i} c_{i} e_{i}\right. \\
& \left.+\left(1-a_{i}\right)\left(1-c_{i}\right) d_{i} e_{i}\right) S_{i} T_{i} \\
& -\left(a_{i} b_{i} c_{i}+a_{i} b_{i} d_{i}-a_{i} b_{i} c_{i} d_{i}+c_{i} e_{i}\right. \\
& \left.-a_{i} b_{i} c_{i} e_{i}+\left(1-a_{i}\right) b_{i}\left(1-c_{i}\right) d_{i} e_{i}\right) S_{i} T_{i}, \\
& x_{15}=\left(1-b_{i}\right)\left(a_{i} c_{i}+a_{i} d_{i}-a_{i} c_{i} d_{i}-a_{i} c_{i} e_{i}\right. \\
& \left.+\left(1-a_{i}\right)\left(1-c_{i}\right) d_{i} e_{i}\right) S_{i} T_{i}, \\
& x_{17}=-\left(c_{i}\left(b_{i}^{\prime}+d_{i}-b_{i}^{\prime} d_{i}\right)+b_{i} d_{i}\left(1-c_{i}\right)\right) S_{i} T_{i}, \\
& x_{18}=-\left(a_{i}\left(b_{i}+e_{i}-b_{i} e_{i}\right)+b_{i}^{\prime} e_{i}\left(1-a_{i}\right)\right) S_{i} T_{i} \text {, } \\
& x_{19}=\left(1-b_{i}\right)\left(1-c_{i}\right) d_{i} S_{i} T_{i} \text {, } \\
& x_{20}=a_{i}\left(1-b_{i}\right)\left(1-e_{i}\right) S_{i} T_{i} \text {. }
\end{aligned}
$$

For $i=0$, we must set $a_{0}=d_{0}=1$ and $c_{0}=e_{0}=0$. These formulae apply to the most general directed $K_{4}$ ladder, and we recover the undirected case by setting $a_{i}^{\prime}=a_{i}, b_{i}^{\prime}=b_{i}$, etc. A missing edge or node is accounted for by setting the relevant reliability to zero, as will be seen in the following section.

\section{ApPliCATION}

Let us apply the results of the preceding section to the architecture represented in Fig. 4. The calculations are straightforward, since we merely have to replace all nonexistent edges and nodes by zero. Does the removal of these network elements drastically change the previous results? Actually, no, even though in the directed case the dimension of the transfer matrix is reduced.

\section{A. Transfer matrices}

In both directed and undirected configurations, we must set $T_{0}=T_{n}=0$ and $b_{i}=0(0 \leq i \leq n)$. This does not change the dimension of the transfer matrix in the undirected case, which remains equal to 5. However, for the directed configuration, 

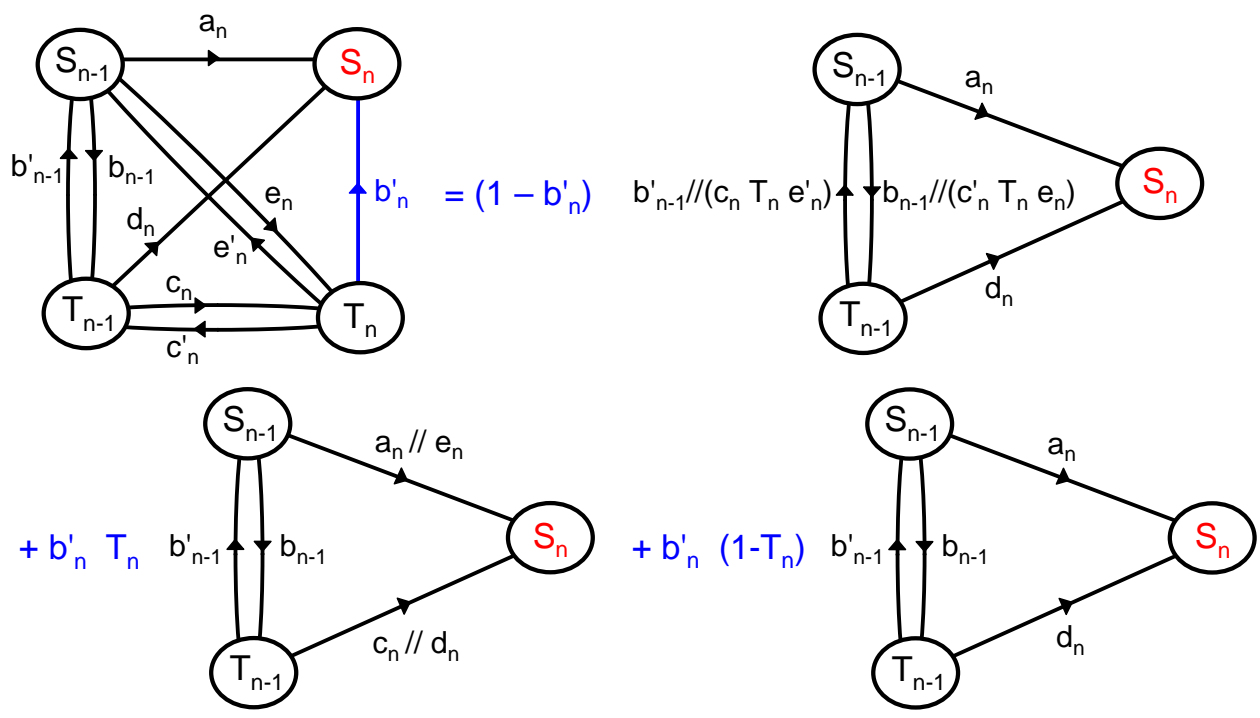

Fig. 3. First step of the decomposition. $a / / b$ corresponds to $a$ and $b$ in parallel, and is therefore equal to $a+b-a b$.

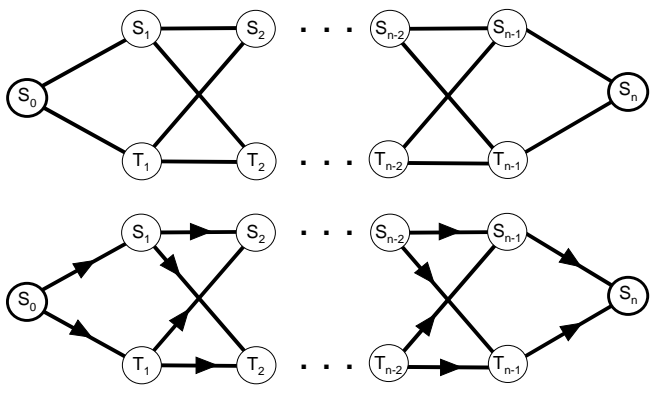

Fig. 4. Architecture discussed in this work ("Échelle Angèle"). The source is $S_{0}$, the destination is $S_{n}$ for both directed and undirected networks.

the further simplification $a_{i}^{\prime}=b_{i}^{\prime}=c_{i}^{\prime}=d_{i}^{\prime}=e_{i}^{\prime}=0$ is such that we can reduce the transfer matrix to a $3 \times 3$ one, namely

$$
\widetilde{M}_{i}=\left(\begin{array}{ccc}
a_{i} S_{i} & d_{i} S_{i} & a_{i} d_{i} S_{i} \\
e_{i} T_{i} & c_{i} T_{i} & c_{i} e_{i} T_{i} \\
-a_{i} e_{i} S_{i} T_{i} & -c_{i} d_{i} S_{i} T_{i} & \chi_{i} S_{i} T_{i}
\end{array}\right)
$$

with $\chi_{i}=a_{i} c_{i}\left(1-d_{i}\right)\left(1-e_{i}\right)+d_{i} e_{i}\left(1-a_{i}-c_{i}\right)$, so that

$$
\widetilde{\mathcal{R}}_{n}=\left(\begin{array}{lll}
1 & 0 & 0
\end{array}\right) \widetilde{M}_{n} \widetilde{M}_{n-1} \cdots \widetilde{M}_{1} \widetilde{M}_{0}\left(\begin{array}{l}
1 \\
0 \\
0
\end{array}\right) .
$$

In many studies, edge reliabilities are considered identical to $p$ in order to provide clues to the general behavior of the connection reliability, while nodes are viewed as perfect (i.e., their reliabilities are set equal to 1). In this work, we keep imperfect nodes with identical reliability $\rho$. We thus have two independent parameters to describe $\operatorname{Rel}_{2}\left(S_{0} \rightarrow S_{n}\right)$, allowing us to better distinguish the contributions of edges and nodes. Using only these two parameters implies that a unique transfer matrix needs be considered. Equations (2) and (5) show that the two-terminal reliability is given by the $n^{\text {th }}$ power of this matrix. For instance, eq. (4) leads to

$$
\widetilde{M}(p, \rho)=\left(\begin{array}{ccc}
p \rho & p \rho & p^{2} \rho \\
p \rho & p \rho & p^{2} \rho \\
-p^{2} \rho^{2} & -p^{2} \rho^{2} & p^{2} \rho^{2}\left(2-4 p+p^{2}\right)
\end{array}\right) .
$$

\section{B. Generating functions}

Because of the intrinsic recursion relation between successive powers of $\widetilde{M}(p, \rho)$, a similar one should hold for the twoterminal reliability. The generating function formalism [49] is a useful way to store all necessary information in a very concise manner. It is defined by

$$
\mathcal{G}(z)=\sum_{n=0}^{\infty} \operatorname{Rel}_{2}\left(S_{0} \rightarrow S_{n}\right) z^{n}
$$

Its calculation is straightforward. Here, $\mathcal{G}(z)$ is necessarily a rational fraction of $z$, namely

$$
\mathcal{G}(z)=\frac{\mathcal{N}(z)}{\mathcal{D}(z)} .
$$

Using eqs. (5) and (6) (for the directed configuration), we compute the first values of $\operatorname{Rel}_{2}\left(S_{0} \rightarrow S_{n}\right)$ for, say, $n$ equal to $2,3,4$, etc., in order to obtain the first terms of the expansion in $z$ of $\mathcal{G}(z)$. We then multiply this truncated expression by the characteristic polynomial of the transfer matrix taken at $1 / z$. The series expansion of this product in the vicinity of $z=0$ leads to $\mathcal{N}(z)$. No determination of eigenvalues or eigenvectors of the transfer matrix is needed.

Having described the method, we limit ourselves to the final, simplified expressions, which should be used for $n \geq 2$, even though they are valid for $n=1$ (both give the first-order term $p \rho^{2} z$, corresponding to the two-terminal reliability between the two nodes $S_{0}$ and $S_{1}$, connected by a single edge). 
1) undirected case:

$$
\begin{aligned}
\mathcal{N}_{u}(z)= & \frac{\rho}{2(1-\rho)}\left(1-p\left(2+2 p-6 p^{2}+3 p^{3}\right) \rho^{2} z\right. \\
+ & \left.2(1-p)^{2}(2-p) p^{3}\left(1-p+p^{2}\right) \rho^{4} z^{2}\right) \\
\mathcal{D}_{u}(z)= & 1-p \rho\left(2+2 p \rho-6 p^{2} \rho+3 p^{3} \rho\right) z \\
& +2(1-p) p^{3} \rho^{3}\left(2-3 p-2 p \rho+6 p^{2} \rho\right. \\
& \left.\quad-4 p^{3} \rho+p^{4} \rho\right) z^{2} \\
& -4(1-p)^{2}(2-p) p^{6}(1-\rho) \rho^{5} z^{3} .
\end{aligned}
$$

2) directed case:

$$
\begin{aligned}
\mathcal{N}_{d}(z)= & \frac{\rho}{2}\left(1-p^{2} \rho^{2}\left(2-4 p+p^{2}\right) z\right), \\
\mathcal{D}_{d}(z)= & 1-p \rho\left(2+2 p \rho-4 p^{2} \rho+p^{3} \rho\right) z \\
& +2 p^{3} \rho^{3}(1-p)(2-p) z^{2} .
\end{aligned}
$$

\section{Analytical expressions of the two-terminal reliabilities}

The two-terminal reliabilities are derived from the partial fraction decomposition of the associated generating functions $\mathcal{G}_{u}(z)$ and $\mathcal{G}_{d}(z)$, because the eigenvalues of the transfer matrix are the inverses of the generating function poles.

1) undirected case: Equations $(9-10$ ) can be further simplified for perfect nodes $(\rho=1)$, because $\mathcal{D}_{u}(z)$ is then of degree 2 in $z$, from which we find

$$
\mathcal{R}_{n}^{(u)}(\rho=1 ; n \geq 2)=a_{+}\left(\zeta_{+}^{(u)}\right)^{n}+a_{-}\left(\zeta_{-}^{(u)}\right)^{n},
$$

with

$$
\begin{aligned}
\zeta_{ \pm}^{(u)}= & \frac{p}{2}\left(2+2 p-6 p^{2}+3 p^{3} \pm \sqrt{\mathcal{A}}\right) \\
a_{ \pm}= & \frac{\mathcal{B} \pm\left(1-4 p+2 p^{2}\right) \sqrt{\mathcal{A}}}{4(1-p)^{2}\left(1-p+p^{2}\right)^{2} \sqrt{\mathcal{A}}} \\
\mathcal{A}= & 4-8 p+36 p^{2}-100 p^{3}+128 p^{4} \\
& -76 p^{5}+17 p^{6} \\
\mathcal{B}= & 2-10 p+38 p^{2}-59 p^{3}+40 p^{4}-10 p^{5} .
\end{aligned}
$$

For $\rho<1$, we would get a sum over three eigenvalues, the analytical expression of which would only be more cumbersome (it will not be given here).

2) directed case: Even if $\rho \neq 1, \mathcal{D}_{d}(z)$ is of degree 2 in $z$. We have again two eigenvalues, so that

$$
\mathcal{R}_{n}^{(d)}(n \geq 2)=\alpha_{+}\left(\zeta_{+}^{(d)}\right)^{n}+\alpha_{-}\left(\zeta_{-}^{(d)}\right)^{n}
$$

with

$$
\begin{aligned}
\zeta_{ \pm}^{(d)}= & \frac{p \rho}{2}\left(2+p \rho\left(2-4 p+p^{2}\right) \pm \sqrt{\mathcal{A}^{\prime}}\right) \\
\alpha_{ \pm}= & \frac{\rho}{4}\left(1 \pm \frac{2-p \rho\left(2-4 p+p^{2}\right)}{\sqrt{\mathcal{A}^{\prime}}}\right) \\
\mathcal{A}^{\prime}= & 4-4 p \rho\left(2-2 p+p^{2}\right) \\
& +p^{2} \rho^{2}\left(2-4 p+p^{2}\right)^{2} .
\end{aligned}
$$

$\zeta_{ \pm}^{(u)}$ and $\zeta_{ \pm}^{(d)}$ are displayed in Fig. 5 for perfect nodes. Obviously, $\zeta_{+}$is nearly always much larger than $\zeta_{-}$, especially when $p$ lies in the vicinity of $1 . \zeta_{+}^{(u)}$ and $\zeta_{+}^{(d)}$ are nearly equal over the whole range $0 \leq p \leq 1$, while the second eigenvalue is much larger in the directed case. Still, as $n$ grows, the contribution of the second eigenvalue should vanish so that the two-terminal reliability exhibits an asymptotic power-law behavior $\mathcal{R}_{n} \propto \zeta_{+}^{n}$, the scaling factor being $\zeta_{+}$, the eigenvalue of largest modulus. Even for $n \approx 10$, this asymptotic limit would already be a good approximation.

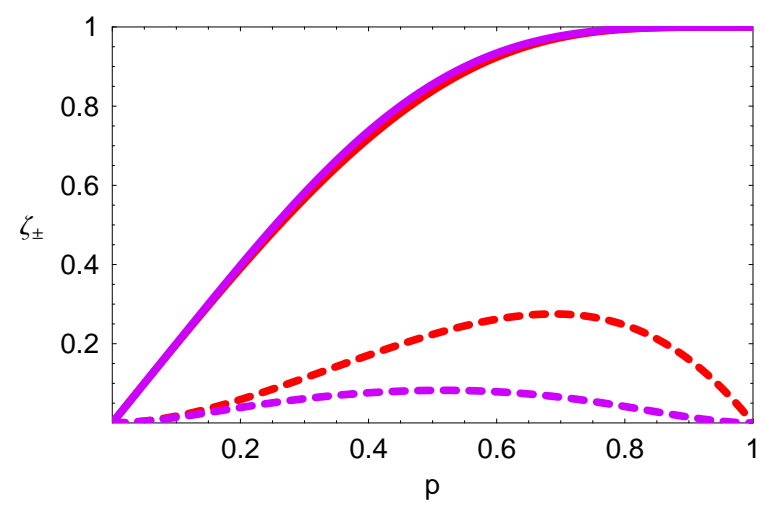

Fig. 5. Variation of $\zeta_{+}$(full line) and $\zeta_{-}$(dashed line) with $p$ for the directed (red curve) and undirected (purple curve) architectures of Fig. 4 with perfect nodes.

\section{Average failure frequency and failure rate}

Steady-state system availability $A$ and failure frequency $\bar{\nu}$ are important performance measures of a repairable system, from which other key parameters such as the mean time between failures, average failure rate, Birnbaum importance, etc. may be deduced [6], [7], [8]. The first calculations of steady-state failure frequencies were based on the inclusionexclusion principle, with adequate failure and repair rates (more generally, the inverses of the mean down and up times) were attributed to each term of the expansion [50], [51]. Several papers have since provided a few simple recipes, describing how $\bar{\nu}$ and the system failure rate $\bar{\lambda}=\bar{\nu} / A$ can then be derived [52], [53], [54], [55]. All these formal calculations boil down to a simple fact: the failure frequency may be derived from $A$ by the application of a linear differential operator [56], [57], [58]:

$$
\bar{\nu}=\sum_{i} \lambda_{i} p_{i} \frac{\partial A}{\partial p_{i}}=\sum_{i} \mu_{i} q_{i} \frac{\partial U}{\partial q_{i}}
$$

where $U=1-A$ is the total unavailability, $p_{i}$ the availability, $q_{i}=1-p_{i}$ the unavailability, $\lambda_{i}$ the failure rate, and $\mu_{i}$ the repair rate of equipment $i$. The expressions obtained in the preceding sections $\left(A \equiv \mathcal{R}_{n}\right)$ make such calculations straightforward by the application of the linear differential operator to each transfer matrix. Good estimates of what happens for large networks $(n \gg 1)$ may be obtained by considering that all links have availability $p$ and failure rate $\lambda$ (assuming nodes are perfect, to keep the discussion simple). If $\mathcal{R}_{n} \approx a_{+} \zeta_{+}^{n}$, the average failure rate $\bar{\lambda}_{n}$ is then given by

$$
\bar{\lambda}_{n}=\frac{\bar{\nu}_{n}}{\mathcal{R}_{n}} \approx \lambda\left(\frac{\partial \ln a_{+}}{\partial \ln p}+n \frac{\partial \ln \zeta_{+}}{\partial \ln p}\right) .
$$




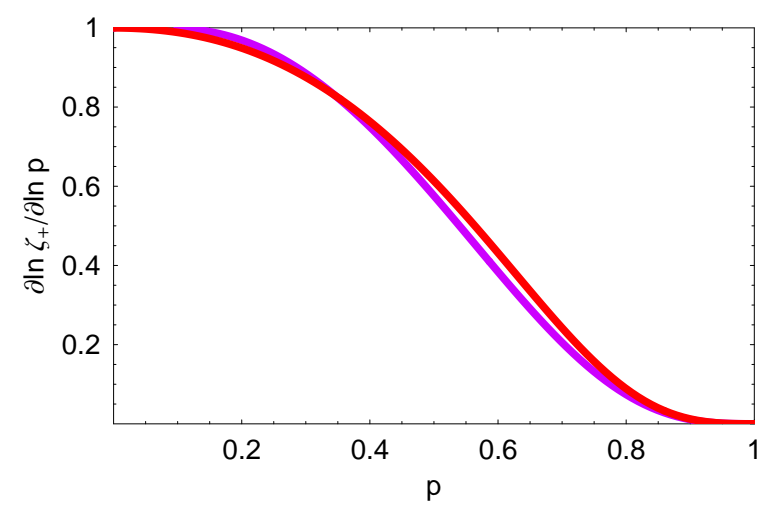

Fig. 6. Variation of $\partial \ln \zeta_{+} / \partial \ln p$ with $p$ for the directed (red curve) and undirected (purple curve) architecture of Fig. 4 with perfect nodes.

The "effective" network can therefore be seen as a series network of $n$ components, having each an availability equal to $\zeta_{+}$and a failure rate $\frac{\partial \ln \zeta_{+}}{\partial \ln p} \lambda$. From Fig. 5 we could expect that the asymptotic failure rate for our case study would be roughly similar for directed and undirected configurations, over the whole range $0 \leq p \leq 1$. This is indeed observed in Fig. 6

\section{E. Zeros of the reliability polynomials}

The structure of the different reliability polynomials may be understood by studying the locations of their zeros in the complex plane. Such a study has been fruitfully performed for chromatic polynomials [59], [60], [61]. In reliability studies, a search for general properties of the all-terminal reliability polynomial $\operatorname{Rel}_{A}(p)$ [36], [37], [38] has brought the BrownColbourn conjecture [39], according to which all the zeros could be found in the region $|1-p|<1$. Although valid for series-parallel graphs, this remarkable conjecture does not strictly hold in the general case (but not by far) [62]. $\operatorname{Rel}_{A}(p)$ is linked to the Tutte polynomial, a graph invariant. It has also been studied extensively by Chang and Shrock for various recursive families of graphs [63], who give the limiting curves where all the zeros converge.

As $n$ grows, the number of complex zeros of the reliability polynomial increases. Because of the matrix transfer property, there is a recursion relation between reliability polynomials corresponding to successive values of $n$. The general problem has been treated by Beraha, Kahane, and Weiss [64]. It may be understood in the following, simplifying way: if the reliability polynomial is of the form $\sum_{i} \alpha_{i} \lambda_{i}(p)^{n}$ (where $\lambda_{i}$ are the eigenvalues of the recurrence), then at large $n$, only the two eigenvalues of greater modules, say $\lambda_{1}$ and $\lambda_{2}$, will prevail, so that the reliability polynomial will vanish when $\left|\lambda_{1}(p)\right|=$ $\left|\lambda_{2}(p)\right|$. This equality defines a set of curves in the complex plane, where all zeros should accumulate in the $n \rightarrow \infty$ limit. A detailed discussion of the convergence to the limiting curves may be found in [61].

The location of the zeros of $\operatorname{Rel}_{2}(p)$ in the complex plane is also worth investigating, even though $\operatorname{Rel}_{2}(p)$ is not a graph invariant. The new twist lies in the extra parameter at our disposal, the node reliability $\rho$, which has a deep impact on the curves to which the zeros of $\operatorname{Rel}_{2}(p)$ converge as $n \rightarrow \infty$. Actually, structural changes occur at critical values of $\rho$, which can be deduced from the expressions of the eigenvalues.

We have displayed in Figs. 7 and 8 the location of complex zeros of the two-terminal reliability polynomials from our case study (the numerical values have been obtained by using MathematicA). The structures of these zeros are quite different for the directed and undirected cases. In both configurations, parts of the real positive axis are indeed the limiting "curve". It turns out that in the undirected case, there is no such real line segment for exactly $\rho_{c_{1}}=\frac{8}{9}$ (the sole intersection of the right-most curve with the real axis lies at $p=\frac{3}{2}$ ). We shall not detail the other critical values for $\rho$. In the directed case, the segment gets closer and "punctures" the curve on the right half-plane for $\rho_{c_{2}} \approx 0.51242$ (a root of a polynomial of degree 10). As $\rho$ further decreases, both structures look like circles plus an extra line segment, which expands as $\rho^{-1 / 3}$. We limit ourselves to the asymptotic values of $\left|p_{\text {circle }}\right|$ (the circle) and $p_{ \pm}$(the endpoints of the segment on the real positive axis). Note the expansion rates are distinct, too.

1) undirected case:

$$
\begin{aligned}
p_{ \pm}^{(u) \rightarrow} & \left(\frac{\sqrt{17}-3}{2 \rho}\right)^{\frac{1}{3}} \pm \sqrt{\frac{34-2 \sqrt{17}}{153}}\left(\frac{\sqrt{17}-3}{2 \rho}\right)^{\frac{1}{6}} \\
& +\frac{2}{51}(23-\sqrt{17}) \\
\left|p_{\text {circle }}^{(u)}\right| \rightarrow & \left(\frac{\sqrt{17}-3}{2 \rho}\right)^{\frac{1}{3}} .
\end{aligned}
$$

2) directed case:

$$
\begin{aligned}
p_{ \pm}^{(d)} & \rightarrow\left(\frac{2}{\rho}\right)^{\frac{1}{3}} \pm \frac{2}{3}\left(\frac{2}{\rho}\right)^{\frac{1}{6}}+\frac{4}{3}, \\
\left|p_{\text {circle }}^{(d)}\right| & \rightarrow\left(\frac{2}{\rho}\right)^{\frac{1}{3}} .
\end{aligned}
$$

\section{CONCLUSION AND OUTLOOK}

The two-terminal reliability of directed networks may also be expressed by a product of transfer matrices, in which each edge and node reliability is exactly taken into account. The size of the transfer matrix should of course increase with the network's "width". This general result could be extended to the all-terminal reliability with nonuniform links [65]. We can now go beyond series-parallel simplifications and look for new (wider) families of exactly solvable, meshed architectures that may be useful for general reliability studies (as building blocks for more complex networks), for the enumeration of selfavoiding walks on lattices, and for directed percolation with imperfect bonds and sites. Since the true generating function is itself a rational fraction, Padé approximants could provide efficient upper or lower bounds for these studies. Moreover, individual reliabilities can be viewed as average values of random variables. Having access to each edge or node allows the introduction of disorder or correlations in calculations [66]. 

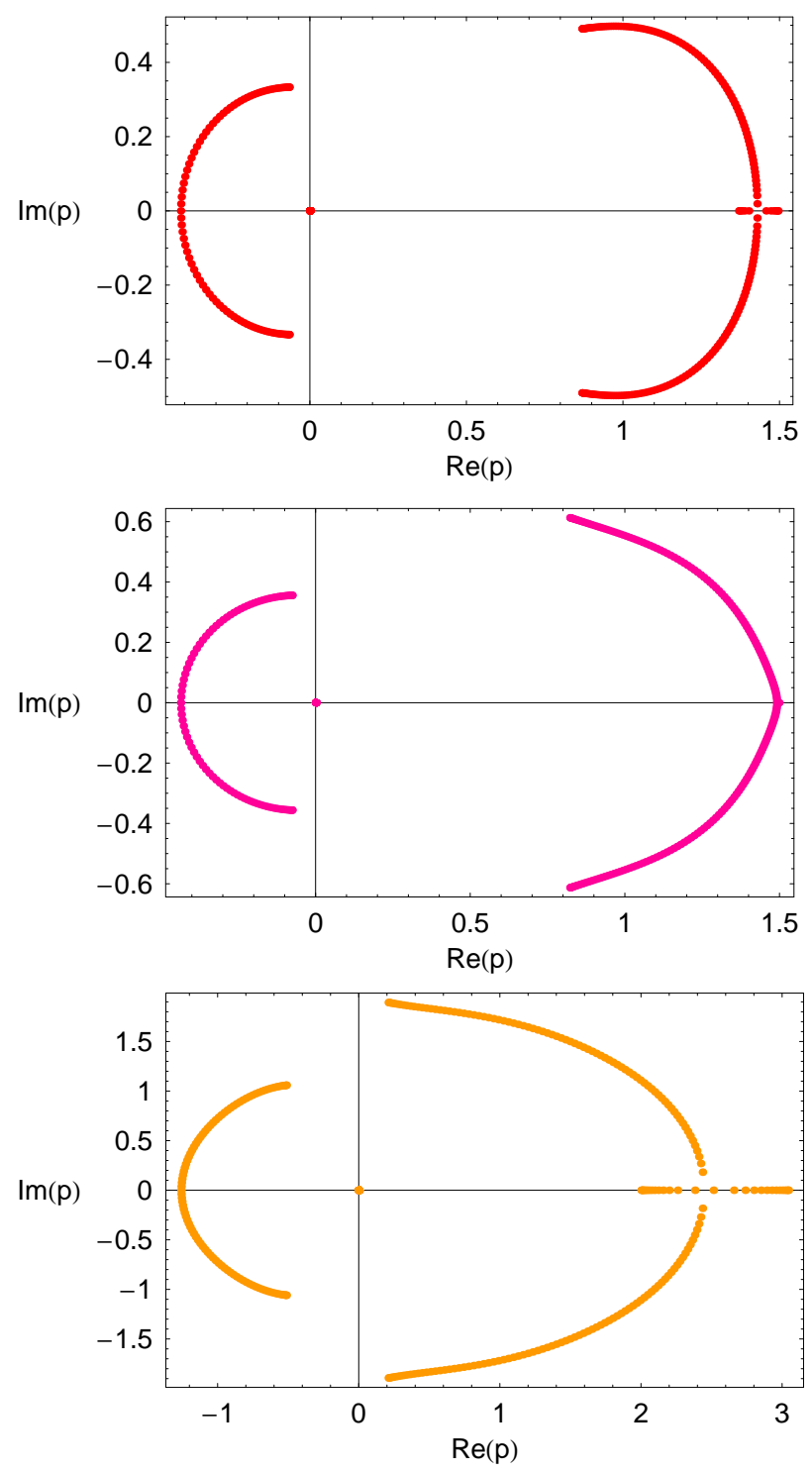

Fig. 7. Location of complex zeros for the undirected ladder, with $n=100$ and $\rho$ equal to $1,0.9$, and 0.1 (from top to bottom).

\section{ACKNOWLEDGMENT}

The author would like to thank Angèle Phu for the architecture described in this work, Éric Gourdin, James Roberts, and Sara Oueslati for helpful discussions, and Olivier Klopfenstein and Benoît Lardeux for very useful suggestions.

\section{REFERENCES}

[1] E. F. Moore and C. E. Shannon, "Reliable circuits using less reliable relays," J. Franklin Institute, vol. 262, pp. 191-208, September 1956; vol. 262, pp. 281-297, October 1956.

[2] M. O. Ball, C. J. Colbourn, and J. Scott Provan, "Network reliability," Handbooks in operations research and management science, volume 7 : Network Models, M. O. Ball, T. L. Magnanti, C. L. Monma, and G. L. Nemhauser (Editors), Amsterdam: Elsevier, 1995, pp. 673-762 (with over 400 references).

[3] R. E. Barlow, F. Proschan, and L. C. Hunter, Mathematical theory of reliability, New York:Wiley, 1965.

[4] C. J. Colbourn, The combinatorics of network reliability, Oxford: Oxford University Press, 1987
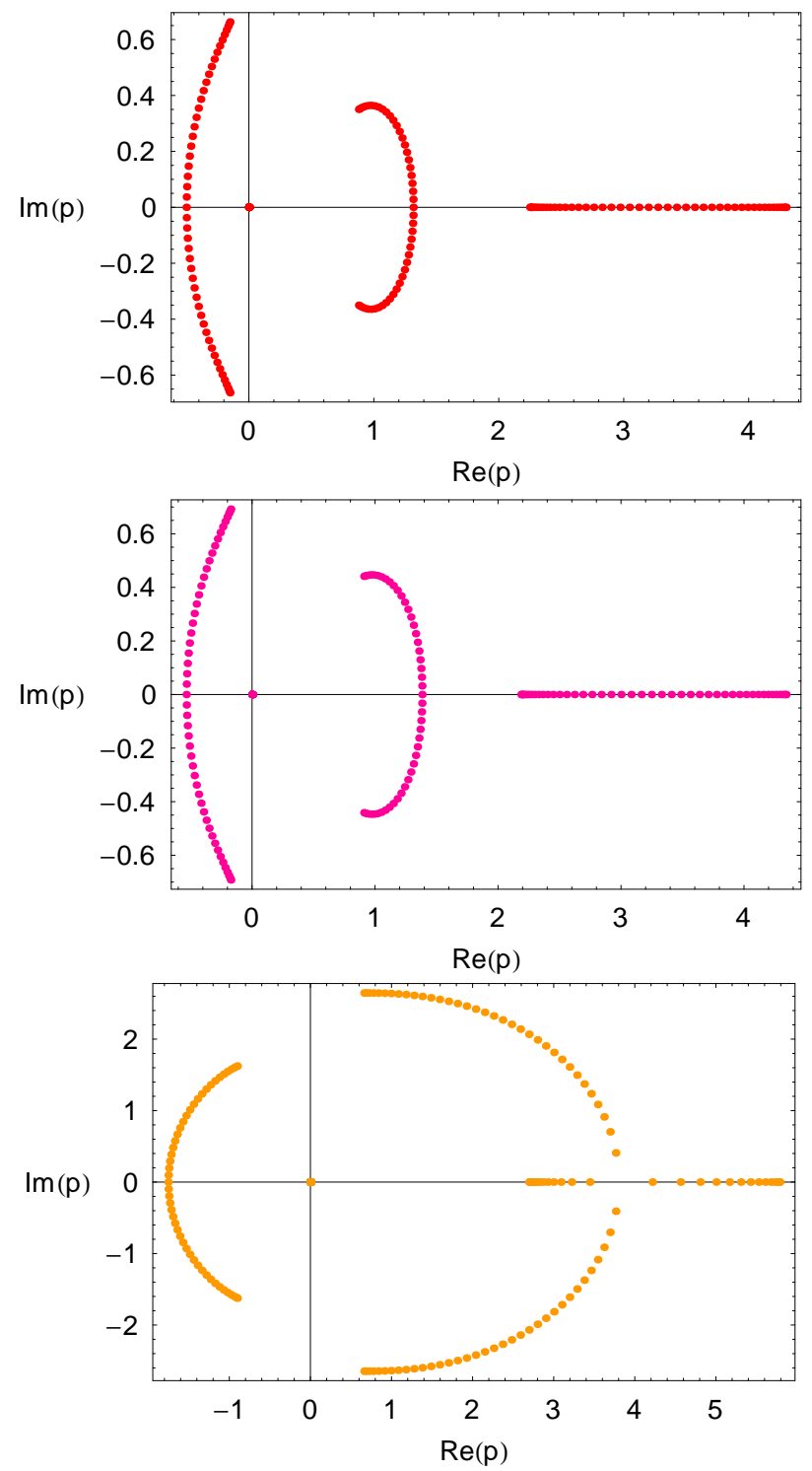

Fig. 8. Location of complex zeros for the directed ladder, with $n=50$ and $\rho$ equal to $1,0.9$, and 0.1 (from top to bottom).

[5] D. R. Shier, Network reliability and algebraic structures, Oxford: Clarendon Press, 1991.

[6] M. L. Shooman, Probabilistic reliability: an engineering approach, New York: McGraw-Hill, 1968.

[7] W. Kuo and M. J. Zuo, Optimal Reliability Modeling: Principles and Applications, Hoboken:Wiley, 2003.

[8] C. Singh and R. Billinton, System reliability modelling and evaluation, London: Hutchinson, 1977.

[9] H. M. AboElFotoh and C. J. Colbourn, "Computing 2-terminal reliability for radio-broadcast networks," IEEE Trans. Reliability, vol. 38 (5), pp. 538-555, 1989.

[10] J. Graver and M. Sobel, "You may rely on the reliability polynomial for much more than you might think," Communications in Statistics: Theory and Methods, vol. 34 (6), 1411-1422, 2005.

[11] D. J. A. Welsh, "Complexity: Knots, Colourings and Counting," London Mathematical Society, Lecture Note Series 186, Cambridge: Cambridge University Press, 1993.

[12] W. P. Dotson and J. O. Gobien, "A new analysis technique for probabilistic graphs," IEEE Trans. Circuits and Systems, vol. 26 (10), pp. 855-865, 1979. 
[13] J. A. Abraham, "An improved algorithm for network reliability," IEEE Trans. Reliability, vol. 28 (1), pp. 58-61, 1979.

[14] A. O. Balan and L. Traldi, "Preprocessing minpaths for sum of disjoint products," IEEE Trans. Reliability, vol. 52 (3), pp. 289-295, 2003.

[15] K. D. Heidtmann, "Smaller sums of disjoint products by subproduct inversion," IEEE Trans. Reliability, vol. 38, pp. 305-311, 1989.

[16] S. Rai, M. Veeraraghavan, and K. S. Trivedi, "A survey of efficient reliability computation using disjoint products approach," Networks, vol. 25 , pp. 147-163, 1995, and references therein.

[17] A. Rauzy, E. Châtelet, Y. Dutuit, and C. Bérenguer, "A practical comparison of methods to assess sum-of-products," Reliability Engineering and System Safety, vol. 79, pp. 33-42, 2003.

[18] S. Soh and S. Rai, "Experimental results on preprocessing of path/cut terms in sum of disjoint products technique," IEEE Trans. Reliability, vol. 42 (1), pp. 24-33, 1993.

[19] J. P. Gadani, "System effectiveness evaluation using star and delta transformations," IEEE Trans. Reliability, vol. 30 (1), pp. 43-47, 1981.

[20] A. Rosenthal and D. Frisque, "Transformations for simplifying network reliability calculations," Networks, vol. 7, pp. 97-111, 1977; errata, Networks, vol. 7, p. 382, 1977.

[21] S.-D. Wang and C.-H. Sun, "Transformations of star-delta and delta-star reliability networks," IEEE Trans. Reliability, vol. 45 (1), pp. 120-126, 1996.

[22] R. Kevin Wood, "A factoring algorithm using polygon-to-chain reductions for computing $K$-terminal network reliability," Networks, vol. 15 , pp. 173-190, 1985.

[23] F. Beichelt and L. Spross, "Bounds on the reliability of binary coherent systems," IEEE Trans. Reliability, vol. 38 (4), pp. 425-427, 1989.

[24] T. B. Brecht and C. J. Colbourn, "Improving reliability bounds in computer networks," Networks, vol. 16, pp. 369-380, 1986.

[25] J. Scott Provan, "Bounds on the reliability of networks," IEEE Trans. Reliability, vol. 35 (3), pp. 260-268, 1986.

[26] G. S. Fishman, "A comparison of four Monte Carlo methods for estimating the probability of $s-t$ connectedness," IEEE Trans. Reliability, vol. 35 (2), pp. 145-155, 1986.

[27] D. R. Karger, "A randomized fully polynomial time approximation scheme for the all terminal reliability problem," SIAM Review, vol. 43 (3), pp. 499-522, 2001.

[28] L. D. Nel and C. J. Colbourn, "Combining Monte Carlo estimates and bounds for network reliability," Networks, vol. 20, pp. 277-298, 1990.

[29] S. Kuo, S. Lu, and F. Yeh, "Determining terminal pair reliability based on edge expansion diagrams using OBDD," IEEE Trans. Reliability, vol. 48 (3), pp. 234-246, 1999.

[30] A. Rauzy, "A new methodology to handle Boolean models with loops," IEEE Trans. Reliability, vol. 52, pp. 96-105, 2003.

[31] F. M. Yeh, S. K. Lu, and S. Y. Kuo, "OBDD-based evaluation of kterminal network reliability," IEEE Trans. Reliability, vol. 51, pp. 443451, 2002.

[32] Fu-Min Yeh, Hung-Yau Lin, and Sy-Yen Kuo, "Analyzing network reliability with imperfect nodes using OBDD," Proceedings of the 2002 Pacific Rim International Symposium on Dependable Computing (PRDC'02), pp. 89-96.

[33] J. Carlier and C. Lucet, "A Decomposition Algorithm for Network Reliability Evaluation,” Discr. Appl. Math., vol. 65, pp. 141-156, 1996.

[34] H. L. Bodlaender, "Discovering Treewidth," technical report UU-CS2005-018, Utrecht University, 2005, and references therein.

[35] J. Galtier, A. Laugier, and P. Pons, "Algorithms to evaluate the reliability of a network," Proceedings of the Workshop on the Design of Reliable Communication Networks 2005 (DRCN'05), pp. 93-100.

[36] M. K. Chari and C. J. Colbourn, "Reliability polynomials: a survey," J. Combin. Inform. System Sci., vol. 22, pp. 177-193, 1997; August 12, 1998 Web version.

[37] C. J. Colbourn, "Some open problems on reliability polynomials," DIMACS technical report 93-28, April 1993; Congressus Numerantium, vol. 93, pp. $187-202,1993$.

[38] J. Oxley and D. Welsh, "Chromatic, flow and reliability polynomials: the complexity of their coefficients," Combinatorics, Probability and Computing, vol. 11, pp. 403-426, 2002.

[39] J. I. Brown and C. J. Colbourn, "Roots of the reliability polynomial," SIAM J. Disc. Math., vol. 5 (4), pp. 571-585, 1992.

[40] E. Hänsler, G. K. McAuliffe, and R. S. Wilkov, "Exact Calculation of Computer Network Reliability," Networks, vol. 4, pp. 95-112, 1974.
[41] W.-J. Ke and S.-D. Wang, "Reliability evaluation for distributed computing networks with imperfect nodes," IEEE Trans. Reliability, vol. 46 (3), pp. 342-349, 1997.

[42] V. A. Netes and B. P. Filin, "Consideration of node failures in networkreliability calculation," IEEE Trans. Reliability, vol. 45 (1), pp. 127-128, 1996.

[43] O. R. Theologou and J. G. Carlier, "Factoring \& reductions for networks with imperfect vertices," IEEE Trans. Reliability, vol. 40 (2), pp. 210 217, 1991.

[44] D. Torrieri, "Calculation of node-pair reliability in large networks with unreliable nodes," IEEE Trans. Reliability, vol. 43 (3), pp. 375-377 and 382, 1994.

[45] C. Tanguy, "Exact solutions for the two- and all-terminal reliabilities of a simple ladder network," unpublished (arXiv:cs.PF/0612143).

[46] C. Tanguy, "Exact solutions for the two- and all-terminal reliabilities of the Brecht-Colbourn ladder and the generalized fan," unpublished (arXiv:cs.PF/0701005).

[47] C. Tanguy, "What is the probability of connecting two points ?," unpublished (arXiv:cs.PF/0612142).

[48] N. Biggs, Algebraic graph theory, 2nd edition, Cambridge: Cambridge University Press, 1993.

[49] R. P. Stanley, Enumerative combinatorics, vol. 1, Cambridge: Cambridge University Press, 1997, chapter 4.

[50] C. Singh and R. Billinton, "A new method to determine the failure frequency of a complex system," IEEE Trans. Reliability, vol. R-23, pp. 231-234, October 1974.

[51] C. Singh, "Tie set approach to determine the frequency of system failure," Microelectron. \& Reliab., vol. 14, pp. 293-294, 1975.

[52] W. G. Schneeweiss, "Computing failure frequency, MTBF \& MTTR via mixed products of availabilities and unavailabilities," IEEE Trans. Reliability, vol. R-30, pp. 362-363, October 1981.

[53] D.-H. Shi, "General formulas for calculating the steady-state frequency of system failure," IEEE Trans. Reliability, vol. R-30, pp. 444-447, December 1981

[54] S. V. Amari, "Generic rules to evaluate system-failure frequency," IEEE Trans. Reliability, vol. 49, pp. 85-87, March 2000.

[55] Y.-R. Chang, S. V. Amari, and S.-Y. Kuo, "Computing System Failure Frequencies and Reliability Importance Measures Using OBDD,” IEEE Trans. Computers, vol. 53, pp. 54-68, January 2004.

[56] W. G. Schneeweiss, "Addendum to: Computing failure frequency via mixed products of availabilities and unavailabilities," IEEE Trans. Reliability, vol. R-32, pp. 461-462, December 1983.

[57] M. Hayashi, "System failure-frequency analysis using a differential operator," IEEE Trans. Reliability, vol. 40, pp. 601-609, 614, December 1991.

[58] A. Druault-Vicard and C. Tanguy, "Exact Failure Frequency Calculations for Extended Systems," unpublished (arXiv:cs.PF/0612141).

[59] N. L. Biggs, R. M. Damerell, and D. A. Sands, "Recursive families of graphs," J. Combin. Theory B, vol. 12 , pp. 123-131, 1972.

[60] N. L. Biggs, "Matrix method for chromatic polynomials," J. Combin. Theory B, vol. 82, pp. 19-29, 2001.

[61] J. Salas and A. D. Sokal, "Transfer matrices and partition-function zeros for antiferromagnetic Potts models. I. General theory and square-lattice chromatic polynomial," J. Statist. Phys., vol. 104, pp. 609-699, 2001; cond-mat/0004330

[62] G. F. Royle and A. D. Sokal, "The Brown-Colbourn conjecture on zeros of reliability polynomials is false," J. Combin. Theory B, vol. 91 (2), pp. 345-360, 2004; arXiv:math.CO/0301199

[63] S.-C. Chang and R. Shrock, "Reliability polynomials and their asymptotic limits for families of graphs," J. Statist. Phys., vol. 112, pp. 10191077, 2003; arXiv:cond-mat/0208538

[64] S. Beraha, J. Kahane, and N. J. Weiss, "Limits of zeros of recursively defined families of polynomials," Studies in Foundations and Combinatorics, Advances in Mathematics Supplementary Studies, vol. 1, G.-C. Rota (Editor), Academic Press, New York, 1978, pp. 213-232.

[65] A. D. Sokal, "The multivariate Tutte polynomial (alias Potts model) for graphs and matroids," Surveys in Combinatorics 2005, London Mathematical Society Lecture Note Series 327, Bridget S. Webb (Editor), Cambridge University Press, Cambridge, 2005, pp. 173-226; arXiv:math.CO/0503607

[66] D. W. Coit, T. Jin, and N. Wattanapongsakorn, "System optimization with component reliability estimation uncertainty: a multi-criteria approach,” IEEE Trans. Reliability, vol. 53 (3), pp. 369-380, 2004. 